\title{
Comparison and Combination of Imaging Techniques for Three Dimensional Analysis of Electrical Trees
}

\author{
Roger Schurch ${ }^{1,2}$, Simon M. Rowland ${ }^{1}$, Robert S. Bradley ${ }^{3}$ and Philip J. Withers ${ }^{3}$ \\ ${ }^{1}$ School of Electrical and Electronic Engineering, University of Manchester, Manchester, M13 9PL, UK \\ ${ }^{2}$ Department of Electrical Engineering, Federico Santa Maria Technical University, Valparaiso, Chile \\ ${ }^{3}$ School of Materials, University of Manchester, Manchester, M13 9PL, UK
}

\begin{abstract}
Imaging of electrical trees has been an important tool for studying the phenomenon. The authors have previously shown that electrical trees can be three-dimensionally (3D) imaged and virtual replicas generated using X-ray Computed Tomography (XCT) or Serial Block-Face Scanning Electron Microscopy (SBFSEM). Here these techniques are evaluated and compared for 3D analysis of electrical trees along with conventional optical methods. A number of types of laboratory created trees showing range of morphologies were grown and examined to delineate the capabilities of each technique. Cross-sectional images and virtual replicas of the electrical trees from XCT and SBFSEM techniques were compared both qualitatively and quantitatively. SBFSEM provides greater detail than XCT, evidenced by imaging smaller sub-branches and when comparing parameters such as the number of tree channels, tree length or tree volume captured. On average, SBFSEM captures almost double the number of tree channels per slice than XCT, and virtual replicas in most of the cases have larger volumes. However, SBFSEM is a destructive technique, which makes the imaging process less reliable than XCT and not suitable for multi-stage of tree growth experiments. For full analysis, a combination of imaging techniques is proposed. Optical methods are used first to monitor tree growth. Then, micro-XCT which provides pixel size down to $\sim 0.4 \mu \mathrm{m}$ with a field of view of around $1 \mathrm{~mm} \times 1 \mathrm{~mm}$, can be used to reveal the overall 3D structure of a normal/mature electrical tree. Nano-XCT can be used to explore in more detail regions of interest, with a pixel size of $\sim 65 \mathrm{~nm}$, but a limited field of view of $65 \mu \mathrm{m}$. Finally, sections of the tree can be analyzed in even greater detail using SBFSEM, which can provide resolutions below $50 \mathrm{~nm}$. Using this approach, a deeper and more complete analysis of the structure of electrical trees can be achieved.
\end{abstract}

Index Terms - Electrical trees, Three-dimensional, 3D, Imaging, Optical, X-ray, XCT, SEM, SBFSEM.

\section{INTRODUCTION}

ELECTRICAL treeing is a long-term mechanism of failure of polymeric high voltage insulation. Electrical trees are artefacts of ageing in polymers that are readily grown in the laboratory under high and divergent electrical stress, and are associated with partial discharge activity. Understanding tree growth is therefore important for a number of reasons; firstly, the successful development of new insulation materials depends upon understanding such long term damage processes; secondly, to enable the interpretation of partial discharge patterns in condition monitoring for asset management. Imaging laboratory-grown electrical trees has been an important tool for studying the phenomenon and obtaining a deeper understanding of how trees develop [1].

Manuscript received on 22 May 2014, in final form 11 September 2014, accepted 6 November 2014
The most common imaging technique is optical microscopy, although detailed features have been examined using scanning electron microscopy (SEM) and transmission electron microscopy (TEM) [2-5]. These latter techniques have the limitation of providing only a two-dimensional (2D) view of a small part of the feature. Optical imaging also often masks any detail in dense tree structures. The authors have shown previously that electrical trees can be three-dimensionally (3D) imaged. Virtual replicas of electrical trees can be created using X-ray computed tomography (XCT) or Serial BlockFace SEM (SBFSEM) $[1,6]$. These techniques also enable the entire 3D structure to be analyzed and provide tools to quantify a tree structure [7].

This paper evaluates and compares the XCT and SBFSEM techniques for $3 \mathrm{D}$ imaging of electrical trees, analyzing the benefits and limitations of each technique and proposing the most appropriate technique, or combination of techniques, depending on the application of interest. To delineate the 
capabilities of each technique a number of types of tree having a range of morphologies, commonly referred to as bush-type and branch-type, were grown and examined.

\section{IMAGING TECHNIQUES}

A range of techniques have been used to image or at least detect the presence of electrical trees including optical methods, electron microscopy, laser scanning confocal microscopy, ultrasound and nuclear magnetic resonance [1]. Conventionally for research purposes, optical methods and SEM are used. However, recently 3D imaging of electrical trees using XCT and SBFSEM techniques have been proposed $[1,6]$. An overview of these techniques is presented below, along with optical methods which are well established.

\subsection{OPTICAL METHODS}

Visible-light microscopy is the most frequently used technique for imaging, because it is widely accessible and relatively inexpensive. However, it only provides a projectedview, often with a narrow depth of field, it is only applicable to translucent materials and its resolution (the closest two points that can be resolved) is limited by the wavelength of visible light. According to the Rayleigh criterion, the maximum resolution is proportional to the ratio between the wavelength and the numerical aperture of the instrument used [8]. Normally practical optical resolution is presently around $0.2 \mu \mathrm{m}$ [9]. In electrical tree imaging, the resolution achieved by optical methods is limited to a few micrometers by the transparency of the polymer and the poor contrast between the electrical tree and the bulk material [1].

\subsection{X-RAY COMPUTED TOMOGRAPHY (XCT)}

$\mathrm{X}$-ray radiography is based on measuring the transmission of X-rays through an object. For XCT, a series of 2D radiographs are taken as the sample is rotated about an axis over at least $180^{\circ}$. These are combined using a reconstruction algorithm to compute a virtual 3D replica of the object (see Figure 1). In conventional X-ray imaging, contrast is obtained from the attenuation of X-rays as they pass through the object [10]. However, when imaging low atomic number materials such as polymers - attenuation (absorption) contrast does not provide sufficient contrast between the material (epoxy say) and the feature (gas filled tubes comprising an electrical tree). As an alternative, contrast can be obtained from the phase shift in the X-rays as they pass through the object. This mode is called 'phase-contrast' [11, 12], and it is well suited to electrical tree imaging.

There are various XCT technologies. Depending on the resolution and consequently, field of view, XCT can be categorized as micro or nano-XCT. The X-ray source can be laboratory-based or synchrotron-based. Both technologies are described in the following sub-sections. The main characteristics of the XCT systems deployed in this work are summarized in Table 1.

\subsubsection{STANDARD LABORATORY X-RAY SOURCE}

The X-ray radiation is created by focusing an electron beam, generated by a filament, on to a target in a vacuum chamber. The X-rays produced have a broad spectrum which is controlled by the applied voltage as well as the target material. Tungsten is a typical target material and it gives rise to characteristic peaks in the spectrum at $8-12 \mathrm{keV}$ and at 57$68 \mathrm{keV}$, with a broad (Bremstrahlung) background.

Modern laboratory projection micro-XCT systems operate in cone beam geometry, in which the resolution of the system is influenced by the detector pixel size as well as the size of the region on the target material from which X-rays are generated (the source spot size). The source spot size limits the resolution for large geometrical magnification, with spot sizes of $\sim 1 \mu \mathrm{m}$ being typical for higher resolution micro-XCT systems. For some systems, the detector pixel size is controlled by using optical magnification between the scintillator and CCD (see Table 1 and Figure 1). Phase contrast can be achieved using the in-line/propagation based method [13], which is manifested as an edge-enhancement around sharp boundaries within the object and which increases with distance of the detector from the object. Few laboratory systems show significant phase contrast which is dependent on the transverse coherence of the illuminating X-rays [14].

Systems with a spatial resolution of less than $\sim 0.5 \mu \mathrm{m}$ are referred to as nano-XCT. Such systems having cone beam geometry are limited to around $200-400 \mathrm{~nm}$ resolution [15]. Higher resolutions (the current limit is $50 \mathrm{~nm}$ ) are achieved

Table 1. Summary of the characteristics of XCT systems with the typical values used for electrical tree imaging.

\begin{tabular}{|c|c|c|c|c|c|c|c|}
\hline XCT system ${ }^{\text {(a) }}$ & Equipment & Objective $^{(b)}$ & $\begin{array}{c}\text { Pixel size } \\
(\mu \mathrm{m})\end{array}$ & $\begin{array}{c}\text { FOV }^{(\mathrm{d})} \\
\left(\mathrm{W} \times \mathrm{Hm}^{2}\right)\end{array}$ & Case study & $\mathrm{X}$-rays & $\begin{array}{c}\text { Typical sample } \\
\text { size required }\end{array}$ \\
\hline \multirow{5}{*}{$\begin{array}{l}\text { Laboratory } \\
\text { Micro-XCT }\end{array}$} & \multirow{2}{*}{ Micro-XCT-400 } & $20 X$ & $\sim 1$ & 1.3 & & \multirow{5}{*}{$\begin{array}{l}\text { Micro-focus tungsten target laboratory source } \\
\text { Spot size: } 7 \mu \mathrm{m} \text { (Micro-400) and } 2 \mu \mathrm{m} \text { (Versa) } \\
\text { Typical voltages: } 60-110 \mathrm{kV} \\
\text { Polychromatic } x \text {-rays with energy }>\sim 10 \mathrm{keV} \\
\text { Peaks in spectrum at } \sim 10-12 \mathrm{keV} \text { and } \sim 60 \mathrm{keV}\end{array}$} & \multirow{5}{*}{$2-3 \mathrm{~mm}$} \\
\hline & & $40 X$ & $\sim 0.5$ & 0.67 & Sample $1^{(\mathrm{e})}$ & & \\
\hline & \multirow{3}{*}{ Versa-510/520 } & $10 \mathrm{X}$ & $\sim 0.8-1$ & $2 \times 2$ & & & \\
\hline & & $20 X$ & $\sim 0.4-0.5$ & $0.9 \times 0.9$ & Sample 2, 3 & & \\
\hline & & $40 X$ & $\sim 0.2-0.3$ & $0.45 \times 0.45$ & Sample 4 & & \\
\hline \multirow{3}{*}{$\begin{array}{l}\text { Laboratory } \\
\text { Nano-XCT }\end{array}$} & Nano-XCT-100 & $20 \mathrm{X}$ & 0.13 & $0.065 \times 0.065$ & Sample 2 & \multirow{3}{*}{$\begin{array}{l}\text { Rotating anode source } \\
\text { Quasi monochrom. at } 8.1 \mathrm{keV} \text { (Nano) and } 5.4 \mathrm{keV} \text { (Ultra) } \\
\text { Quasi-parallel beam }\end{array}$} & \multirow{3}{*}{$<0.8 \mathrm{~mm}$} \\
\hline & \multirow{2}{*}{ Ultra-810 } & $20 X$ & 0.064 & $0.065 \times 0.065$ & Sample 4 & & \\
\hline & & $40 X$ & 0.032 & $0.032 \times 0.032$ & & & \\
\hline \multirow{2}{*}{$\begin{array}{l}\text { Synchrotron } \\
\text { Micro-XCT }\end{array}$} & \multirow{2}{*}{$\begin{array}{l}\text { Diamond Light } \\
\text { Source - I13 }\end{array}$} & $4 \mathrm{4X}$ & 1.1 & $4.5 \times 3.0$ & & \multirow{2}{*}{$\begin{array}{l}\text { 9-15 keV/ monochromatic or polychromatic } \\
\text { Parallel beam, high brilliance, coherent }\end{array}$} & \multirow{2}{*}{$2-3 \mathrm{~mm}$} \\
\hline & & $10 \mathrm{X}$ & $0.45^{(\mathrm{f})}$ & $1.8 \times 1.2$ & Sample 3 & & \\
\hline
\end{tabular}

(a) Lab. XCT are Zeiss Xradia systems. (b) Not all the available objectives are presented here, only those typically used for electrical trees. For nano-XCT the zone plate objective is given. (c) For lab. Micro-XCT: cone beam geometry, the effective pixel size depends on the optical and geometrical magnifications, the latter given by the ratio of source-to-detector distance and source-to-sample distance (d) Field of View (FOV) given by multiplying the effective pixel size by sensor dimensions. (e) Scanned with binning 2 (resulting double the pixel size), to ensure reasonable scan time. (f) For the time of the scan for Sample 3, the pixel size was $0.365 \mathrm{um}$. 


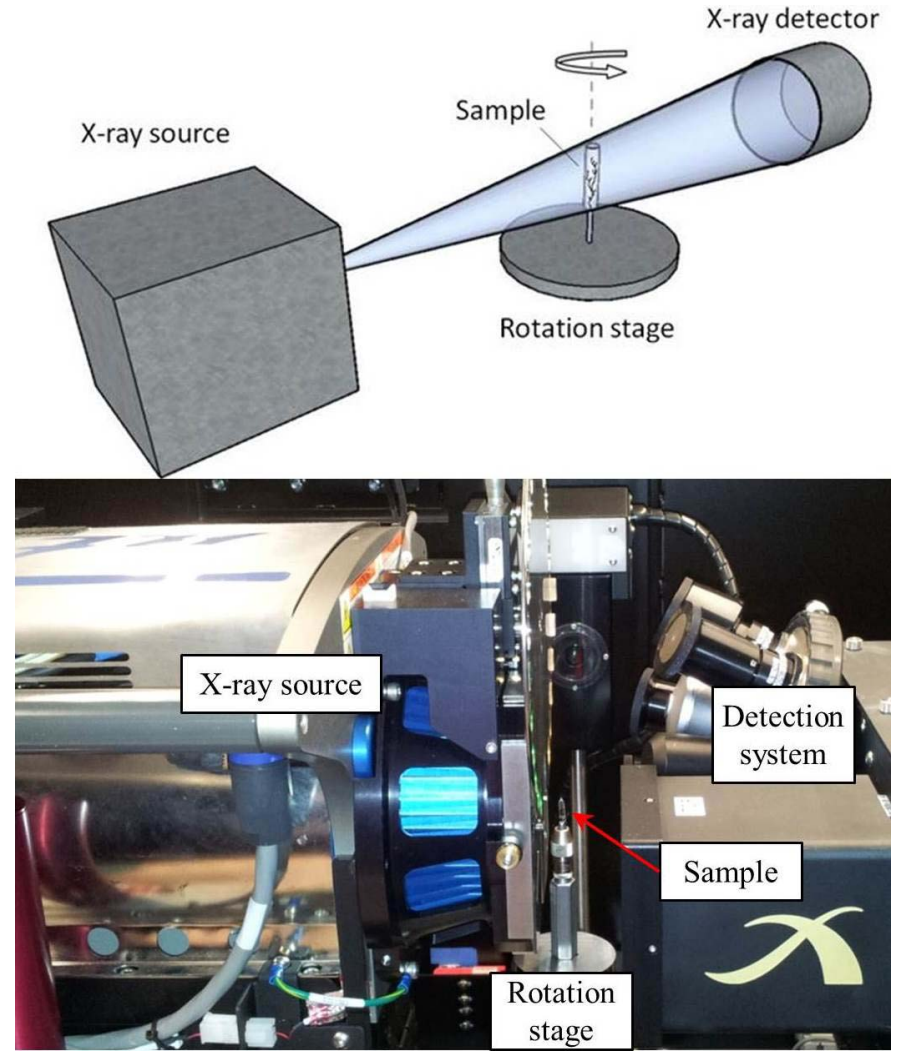

Figure 1. Top: XCT principle. Bottom: Actual XCT setup for micro-XCT. The detection system incorporates optical magnification of a scintillator to achieve sub-micron pixel sizes

using an X-ray microscope design, in which Fresnel zone plates or other optical elements are used as objective lenses [15]. Because the optical elements are usually wavelength dependent, microscope designs make use of a narrow range of $\mathrm{X}$-ray energies, so that the illumination can be considered to be monochromatic as well as quasi-parallel. Zernike phase contrast can be achieved with a phase ring placed in the back focal plane of the objective lens, together with hollow cone illumination [16].

\subsubsection{SYNCHROTRON X-RAY SOURCE}

Large scale synchrotron facilities offer X-rays with high flux, and high coherence, with the ability to tune the X-ray spectrum from a broad spectrum to a monochromatic beam. $\mathrm{X}$-rays are produced when electrons in the storage ring are forced to move in curved paths by bending magnets or insertion devices. Monochromatic X-rays are advantageous since their wavelength can be specifically tuned to achieve greater absorption contrast compared to a broad spectrum source (such as laboratory sources). The high flux enables rapid tomography data collection, so that dynamic experiments can be conducted on the sub-second time scale [17]. The parallel beam geometry also facilitates in-situ experiments since a large distance between sample and detector can be maintained without loss of intensity. Submicron imaging can be achieved with high resolution detectors, which employ optical magnification of scintillator screens. Phase contrast can be obtained via the in-line method or via a variety of other techniques. Sub $30 \mathrm{~nm}$ spatial resolutions can be achieved with X-ray microscope designs, similar to that employed for laboratory nano-XCT [15].

\subsection{SERIAL BLOCK FACE SCANNING ELECTRON MICROSCOPY (SBFSEM)}

\subsubsection{CONVENTIONAL SEM}

SEM is a well-established technique for imaging the surface of materials for identification of morphology/topography. In a SEM a beam of electrons is scanned across the surface of the specimen. The incident beam interacts with the surface of the sample and signals are emitted as a result of these interactions; these emitted signals are collected to form an image. The main information is carried by the 'secondary electrons', which are produced by inelastic collisions of incident primary electrons with the sample [18]. These low energy secondary electrons provide information about the topography or surface structure of the sample, since they are ejected from the orbitals of the atoms within a shallow depth $(<2 \mathrm{~nm})$ of the material surface [19]. Traditionally, for SEM imaging of non-conducting samples, metallization of the surface is needed and the scan is carried out under high vacuum, to avoid sample charging and obtain better resolution respectively.

\subsubsection{LOW VACUUM SEM AND SBFSEM}

Unlike in the standard high vacuum case, when imaging wet and/or insulating materials, the so-called 'environmental' (or 'low-vacuum') SEM can be used [18]. By maintaining a gaseous environment around the sample, low vacuum SEM allows imaging of non-conducting surfaces with only a small loss in image quality [18]. This removes the need of metalizing the polymer surface and thus enables their automated serial sectioning inside the chamber to reconstruct 3D structures.

In SBFSEM, the 3D set of data is acquired by automated serial sectioning (microtome) combined with block-face imaging of the sample, inside a chamber of a low-vacuum SEM [20]. A picture of the equipment is shown in Figure 2.

\section{CASE STUDIES}

A number of sample trees of various morphologies in different polymeric systems have been selected to delineate the capabilities of each imaging technique. The general process for electrical tree analysis consisted of experimental sample preparation, electrical tree formation, machining the tree-containing sample to a suitable size for scanning, image acquisition, and finally post-processing of the data which includes image segmentation and quantitative calculations. These processes are now reviewed in detail:

Samples were prepared using the conventional point-toplane configurations with a gap of $2 \mathrm{~mm}$ between the needle tip (high voltage electrode) and the bottom of the sample (grounded). Electrical trees were grown by applying an AC high voltage (typically around $10 \mathrm{kVrms}$ ) until the desired tree length was reached, the process being monitored with an optical camera. Afterwards, the samples were machined to create a 2-3 mm diameter cylinder of polymer containing the tree to be imaged by the XCT scan, still with the needle inserted. The needle was used to hold the sample and locate the feature in the radiographs, as can be seen in Figure 3. Micro XCT was carried out using three Zeiss Xradia 


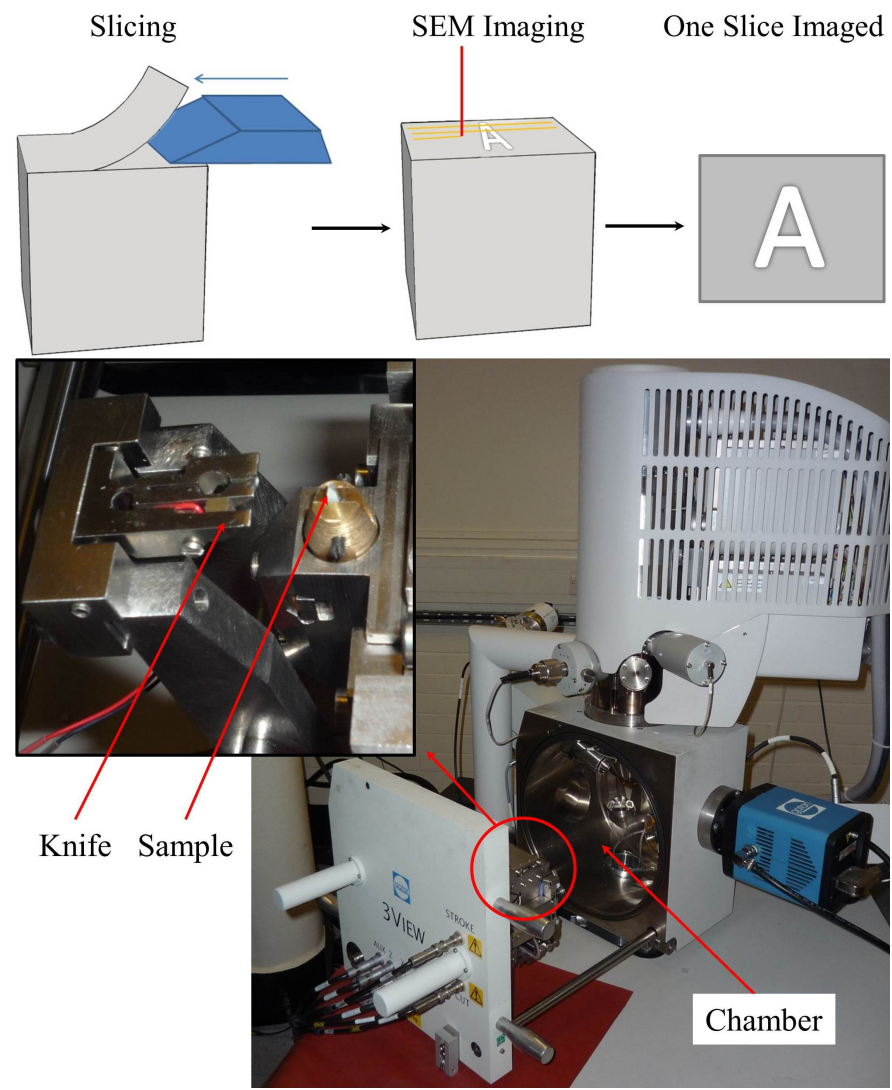

Figure 2. Top: SBFSEM process schematic. Bottom: SBFSEM equipment.

laboratory systems, namely the microXCT-400, Versa 510 and Versa 520, as well as the synchrotron beamline I13 at Diamond Light Source (Figure 3). Nano-XCT was carried out using the Zeiss Xradia nanoXCT-100 and the Zeiss Xradia Ultra 810 laboratory X-ray microscope. In the cases that the SBFSEM technique was used, the small cylinder (already scanned using $\mathrm{XCT}$ ) was machined further to create a cube, containing the feature, with edges less than $1 \mathrm{~mm}$. The needle was removed to prevent damage to the microtome knife. The SBFSEM process was performed using a Gatan 3 View ${ }^{\circledR}$ system housed within an FEI Quanta FEG 250 instrument [21].

The output of the XCT scans is a set of projections (radiographs) taken from different incident angles from which, through the reconstruction process, a stack of images (cross-section slices) is obtained. In contrast, in SBFSEM the automatic slicing process directly provides the stack of images (slices) that represent cross-sections of the electrical tree feature. In both methods, these slices are in grey levels, where different pixel intensities represent different materials (XCT and SBFSEM) or surface topological information (SBFSEM only). From this stage, the model creation procedure follows the same route for both imaging techniques. The pixel size of the images relates to the resolution of the scan and the dimensions to the field of view captured. Using the gray-level information, the $3 \mathrm{D}$ image is segmented, which is the process whereby the feature (electrical tree) is extracted and labelled, and finally, the 3D model (the virtual replica) is generated. Segmentation was carried out using Avizo image processing software. With the segmented model created, the structure of the electrical tree

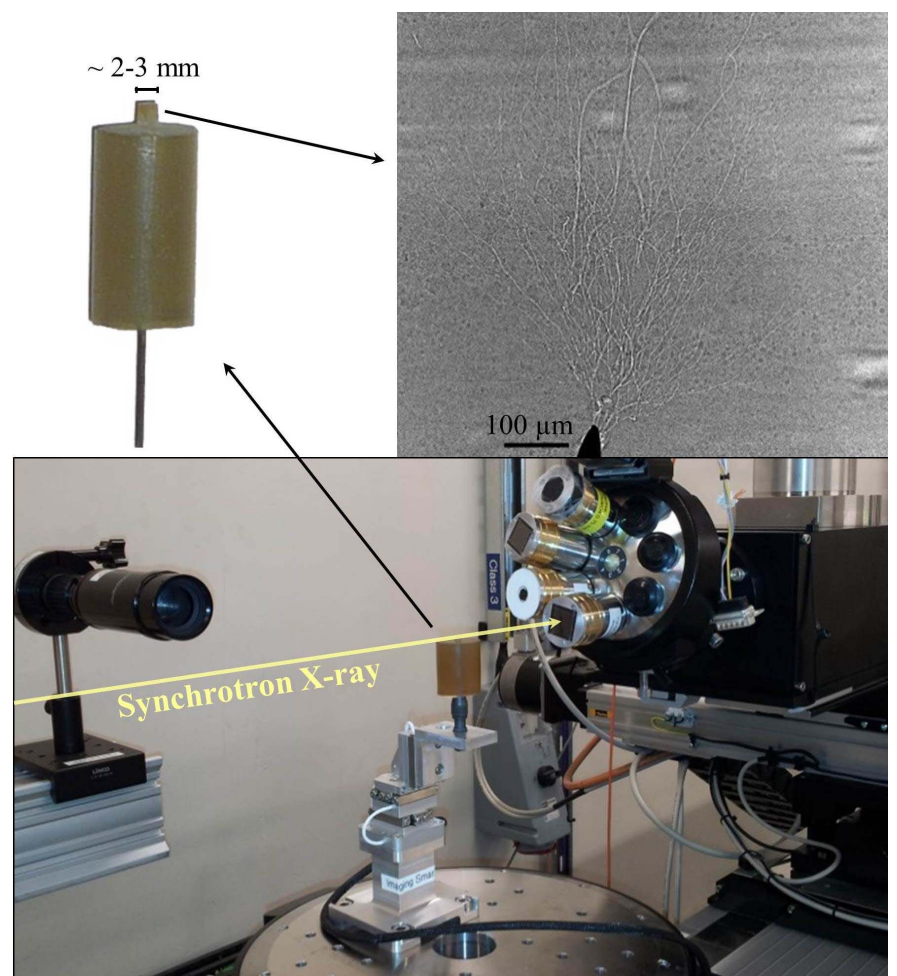

Figure 3. Top-left. Sample with a size of $\sim 2-3 \mathrm{~mm}$ in area to be imaged. Topright: Radiograph and needle tip as reference point (Sample 3). Bottom: Setup for micro-XCT on I13 at the Diamond Light Source Synchrotron.

can be quantified and characterized [7, 22], using software such as Avizo, ImageJ and Matlab.

The following sections describe the samples analyzed in this comparison study, giving experimental details and outlining the results (virtual replicas) generated. Samples 1 and 2 have been reported previously $[1,6,7]$, but are reexamined here from a technique comparison viewpoint. Recorded videos of tree growth, animations of cross-section slices along the progression of the tree and animations of virtual replicas generated are provided in [23].

\subsection{SAMPLE 1: BUSH TREE}

A bush-type electrical tree was studied using micro-XCT and SBFSEM. The electrical tree was created in a flexible transparent polymer (Norland $\AA$ Optical Adhesive 61) with an acupuncture needle (Hwato $\AA 0.35 \mathrm{~mm}, \sim 5 \mu \mathrm{m}$ tip radius). Details of the experiment are provided in [1] and [6]. From the

micro-XCT scan, a virtual 3D image of the tree was reconstructed comprising 512 slices with a voxel (volumetric pixel) size of $1.07 \mu \mathrm{m}$ from which 356 slices (a length of $381 \mu \mathrm{m}$ ) were selected for detailed analysis. In the SBFSEM case, due to technical problems related to sections been drawn into the final aperture and distorting the beam, parts of the data were corrupted and the information of those sections was lost. Because of this, two separated regions of the electrical tree were imaged. The slice thickness was $0.2 \mu \mathrm{m}$ and the pixel size was also $0.2 \mu \mathrm{m}$. The first region comprised 1273 cross-sections of the tree, equivalent to around $255 \mu \mathrm{m}$ from the tree-tips down. The second region, separated by $34 \mu \mathrm{m}$ from the first, comprised 1567 slices (around $313 \mu \mathrm{m}$ ). The location of the sections with respect of the needle tip was unknown because the section close to the needle tip was also 

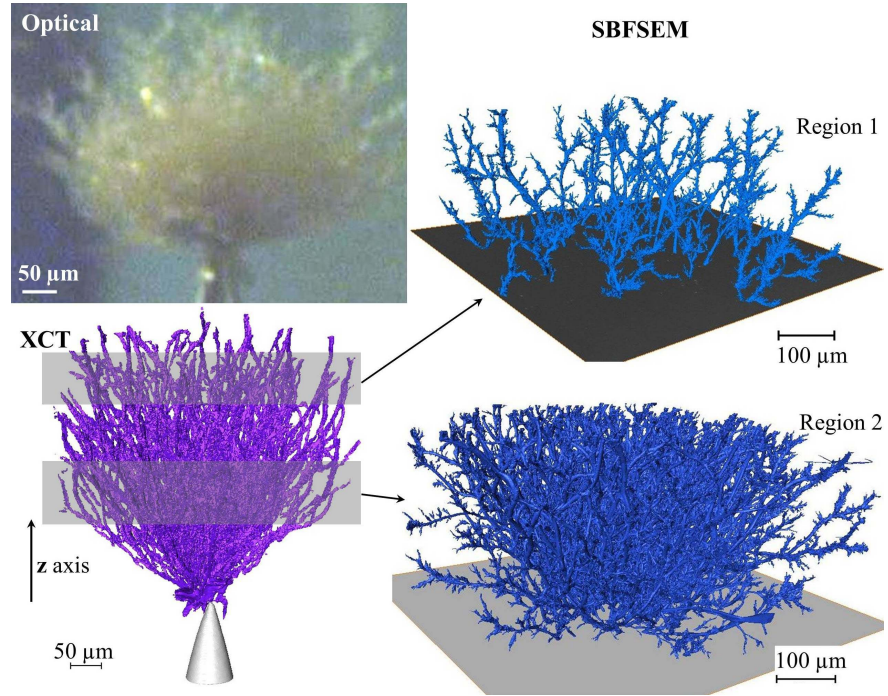

Figure 4. Optical image and 3D renderings of Sample $1-$ Bush tree. The two sections of the SBFSEM are presented on the right with arrows indicating their approximate location in the XCT model shown at the bottom left.

lost, and thus, the reference was lost. The scan time for regions 1 and 2 were $19 \mathrm{~h}$ and $23 \mathrm{~h}$, respectively. Representations of the models generated are shown in Figure 4.

\subsection{SAMPLE 2: SMALL BRANCH TREE}

A small branch-type electrical tree was scanned by the nanoXCT technique (Nano-XCT-100 microscope), micro-XCT (Versa-510) and SBFSEM. The electrical tree was created in a

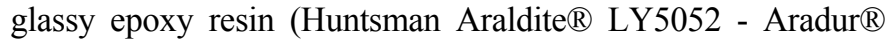
HY5052) using the same needle type as for Sample 1. The applied voltage was maintained only a few seconds after an electrical tree was perceived through the optical camera. The resulting feature was an electrical tree of less than $100 \mu \mathrm{m}$ length. The results from the micro-XCT scan have been reported in [7]. To ensure reasonable nano-XCT scan times ( $\sim 45 \mathrm{~h}) 2 \times 2$ pixels were binned (merged) in the reconstruction to give less noisy slices each having $204 \times 256$ pixels, with a pixel size of $0.26 \mu \mathrm{m}$. The images were noisy

since the physical size of the sample was not as small as required for nano-XCT scans $(\sim 30$ times greater than the field of view), providing a poor signal-to-noise ratio in the given scan time. The virtual replica generated using nano-XCT is shown in Figure 5 and compares unfavorably with the SBFSEM image; as a result the model was not used for quantification.

The micro-XCT scan took $22 \mathrm{~h}$, during which 1501 radiographs were taken over $360^{\circ}$. The voxel size of the reconstruction was $0.37 \mu \mathrm{m}$, with the entire tree being contained within 160 slices (equivalent to $59 \mu \mathrm{m}$ ). The entire tree was also captured using SBFSEM. The operating conditions were set to an accelerating voltage of $5 \mathrm{kV}$ and a pressure of $60 \mathrm{~Pa}$. The slice thickness was $150 \mathrm{~nm}$ with a lateral pixel size of $75 \mathrm{~nm}$. The serial sectioning and imaging took around $7 \mathrm{~h}$ and a total of 456 slices extending over $68 \mu \mathrm{m}$ were selected for model generation. The original images were cropped to $1050 \times 1140$ pixels. Virtual replicas using both methods are depicted in Figure 5, including magnified views of specific branches confirming the greater level of detail expected of the SBFSEM.

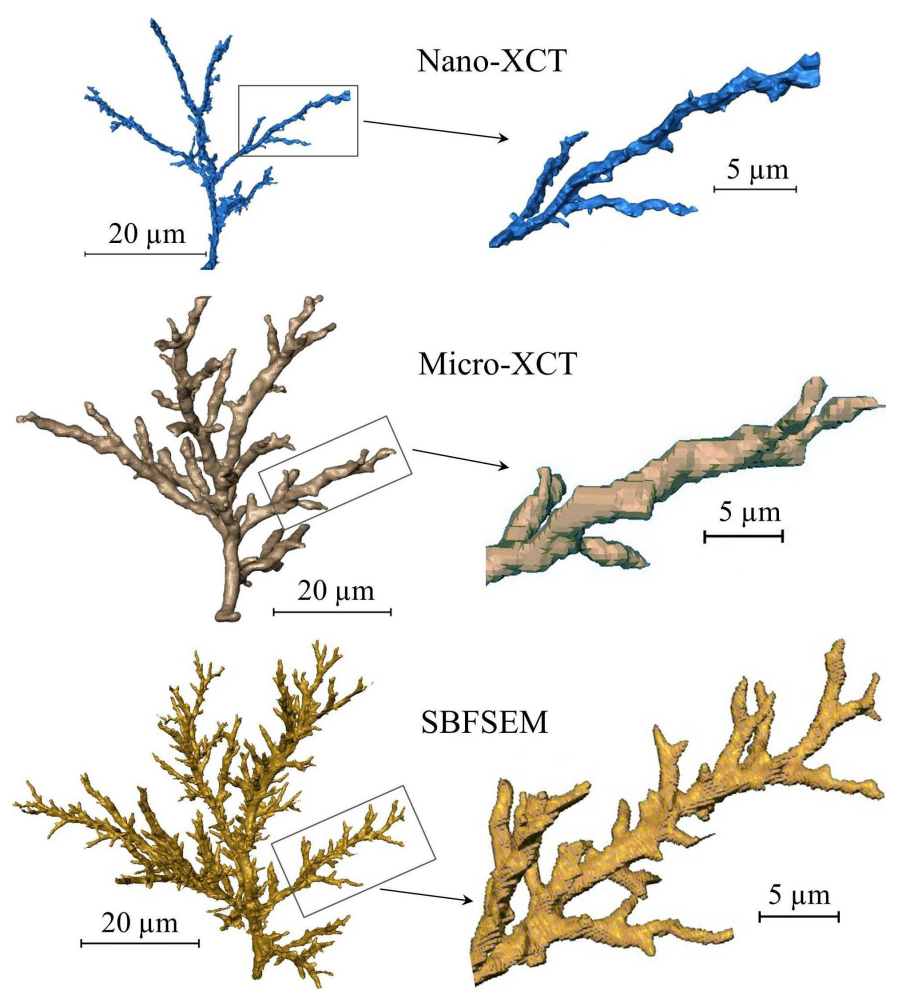

Figure 5. 3D renderings of Sample 2 - Small branch tree. Images on the right are magnified versions of selected branches (highlighted with a box) from the models on the left.

\subsection{SAMPLE 3: LOW DENSITY BUSH TREE}

A low density bush-type tree was selected to compare two micro-XCT systems: the beamline I13 at the Synchrotron Diamond Light Source and the Xradia Versa-510 (laboratorybased XCT). The electrical tree was created using the same transparent polymer as for Sample 1 with an acupuncture needle (Hwato $\AA 0.16 \mathrm{~mm}, \sim 5 \mu \mathrm{m}$ tip radius). The sample was stressed under $8.5 \mathrm{kVrms} 50 \mathrm{~Hz}$ for a period of $3 \mathrm{~h}$.

For the synchrotron scan, the highest magnification available was deployed, giving the smallest pixel size $(0.365$ $\mu \mathrm{m})$. A total of 1001 projections were taken over $180^{\circ}$ in a period of 4-5h. During the scan, the sample moved as it rotated. This unexpected 'wobble' was corrected for by tracking the tip of the needle through the series of radiographs using image correlation and then aligning the radiographs prior to reconstruction. The reconstruction provided images of $4000 \times 4000$ pixels and 2300 slices images (equivalent to $840 \mu \mathrm{m}$ length) were selected for model generation.

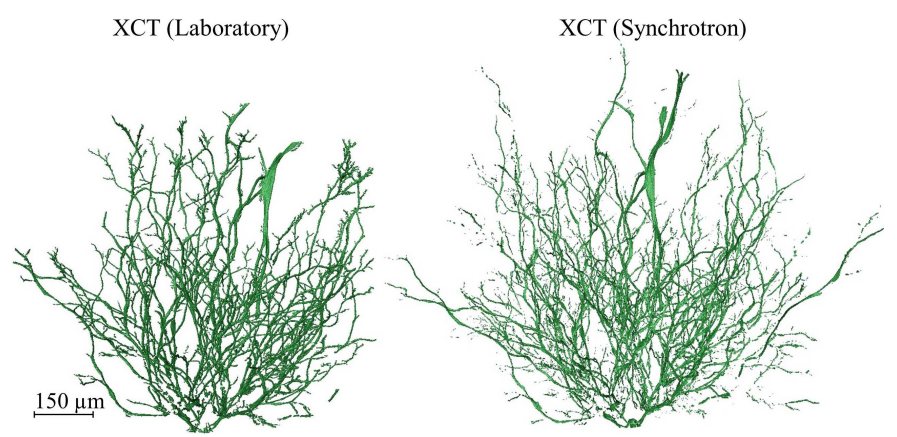

Figure 6. 3D renderings of Sample 3 - Low density bush tree. 
Using laboratory-based micro-XCT, 1301 radiographs were taken over $360^{\circ}$ in a period of $23 \mathrm{~h}$. As result, after reconstruction, 1589 slices (equivalent to $720 \mu \mathrm{m}$ length) were selected with dimensions of $1912 \times 1944$ pixels and $0.454 \mu \mathrm{m}$ voxel size. Images of the $3 \mathrm{D}$ models generated using synchrotron and laboratory-based XCT are depicted in Figure 6.

\subsection{SAMPLE 4: SMALL DENSE BUSH TREE}

A small-dense bush tree was scanned using three imaging systems (instruments): micro-XCT (Versa-520), nano-XCT (Ultra-810) and SBFSEM. In an effort to create a small-dense bush tree, a flexible epoxy (Huntsman Araldite ${ }^{\circledR}$ CY221 Aradur ${ }^{\circledR}$ HY2966) with an Ogura needle (3 $\mu \mathrm{m}$ tip radius) was used. The sample was stressed for 40 minutes at around $10{ }^{\circ} \mathrm{C}$ at $10-12 \mathrm{kVrms}, 50 \mathrm{~Hz}$. The resulting tree was a small dense bush tree, of around $100 \mu \mathrm{m}$ diameter, as can be seen in the optical image shown in Figure 7. For micro-XCT, the scanner was used in 40X magnification and 2001 radiographs were taken over a period of almost $10 \mathrm{~h}$, with a pixel size of $0.232 \mu \mathrm{m}$. A total of 463 slices (equivalent to $108 \mu \mathrm{m}$ of tree length) were selected from the reconstruction for segmentation of the 3D model being shown in Figure 8. The internal structure of this model is observed by virtual slicing of the replica: 20 slices equivalent to $3.5 \mu \mathrm{m}$ are shown on the right hand side of Figure 8.

Prior to the nano-XCT scan, the sample was machined down to size $<1 \mathrm{~mm}$. Over a period of $24 \mathrm{~h}, 721$ radiographs were taken, with a pixel size of $0.064 \mu \mathrm{m}$. In this mode, the nano-XCT machine provides a field of view of $65 \mu \mathrm{m} \times 65 \mu \mathrm{m}$. The reconstructed volume comprised 1022 slices, equivalent to $65 \mu \mathrm{m}$ of length. Given the limited field of view provided, the scan could not capture the entire tree and therefore, no 3D model was generated, because it would represent the tree cropped into a cylindrical shape. Also, the signal-to-noise ratio of the

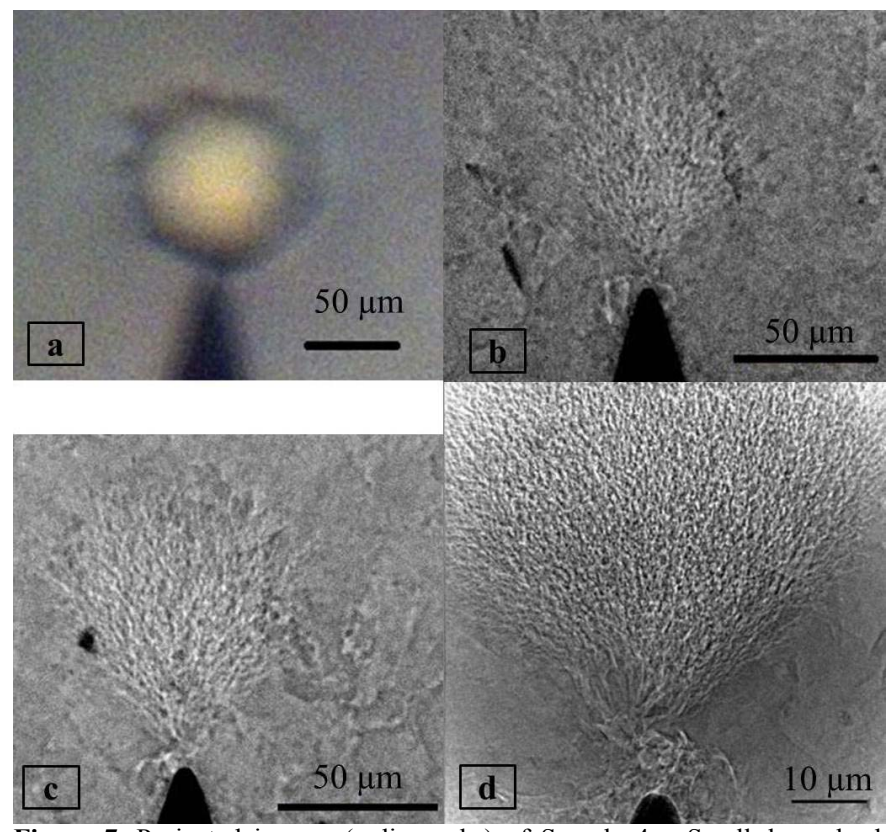

Figure 7. Projected images (radiographs) of Sample 4 - Small-dense bush tree. a. Optical. b. Micro-XCT 20X objective c. Micro-XCT 40X objective d. Nano-XCT reconstructed images was not good enough to successfully segment the electrical tree.

Before applying the SBFSEM method, the sample was reembedded it in epoxy to make it more stable for slicing. The needle was removed, and in this case, the slicing was started from the needle tip, each subsequent slice being further through the tree, making it easy to locate the tree. The scan comprised 1616 slices taken over a period of around $20 \mathrm{~h}$. The accelerating voltage was $5 \mathrm{kV}$ and the pressure $72 \mathrm{~Pa}$. The pixel size was set to approximately $50 \mathrm{~nm}$ with a slice thickness of $100 \mathrm{~nm}$. It was observed that the electron beam softened the epoxy resin making more difficult to maintain the integrity of the specimen during slicing, therefore, a shorter beam dwell time (a faster scan beam movement) was set to reduce this softening. As a result, the contrast obtained was sub-optimal hindering the image segmentation process. The resulting images (bottom of Figure 9) confirm the dense architecture of the tree and shows two distinct areas. In the outer area (brighter region in the bottom right image) individual tree channels can be resolved, by contrast the inner area (darker region) seems to be highly degraded such that individual branches are either not present or cannot be resolved. This issue is discussed in the following sections.

\section{COMPARATIVE ANALYSIS OF THE IMAGING TECHNIQUES}

In the following sections the imaging techniques are compared based on the level of detail revealed by visual inspection and quantitatively by counting the number and mean diameter of tree channels, and the volume and length of tree captured in each case. The complexity of sample preparation and speed of imaging for each technique is also contrasted. It should be noted that different polymers, stress times, image acquisition instruments and scan settings were used (see Table 2), and therefore, a direct comparison between the samples is difficult. Nevertheless, the imaging techniques can be compared for each sample taking into consideration that datasets do not have the same pixel size and the scan settings were optimized to obtain good contrast in all cases.

\subsection{VISUAL ASPECTS}

The initial visual inspection shows that the XCT representations are in accordance with the visual impressions obtained by optical microscopy, as can be seen for Sample 1 (Figure 4) and for Sample 4 (Figures 7 and 8).
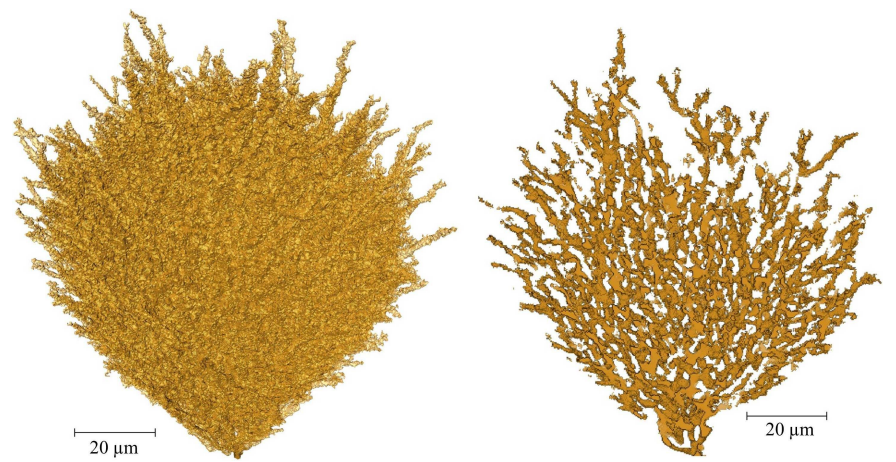

Figure 8. Virtual replica of Sample 4 using micro-XCT (left). Right: internal structure of the dense bush tree shown on the left (obtained displaying 20 slices located in the middle of the tree. Slices in z direction, see Figure 4). 
Comparing the 3D models created using XCT and SBFSEM, it is clear that SBFSEM is able to capture finer details. For example, in Figures 4 and 5 it can be seen that branches from SBFSEM model contain smaller sub-branches (side-branches) than the model from XCT. A clearer view of this is shown in the enlarged branch depicted in Figure 5.

One feature of both techniques is the ability to inspect the internal branches of an electrical tree. This is especially important when analyzing bush-type electrical trees. For example, optical images of bush trees seen in Figures 4 and 7 show dense bush trees that cannot be resolved satisfactorily, at least in the main body of the tree. However, cross-sectional images reveal the internal structure of the trees, as shown in Figure 9. Comparing the cross-sections of both techniques, the greater level of detailed that SBFSEM can provide is evident.

In the case of Sample 4 (small-dense bush tree), the SBFSEM suggests that the inner kernel of the tree is a highly degraded area where individual tubes are either not present or difficult to differentiate. As to whether this could be an artefact of the destructive slicing method can be answered by examination of the XCT images which were acquired nondestructively and are shown in Figure 9. This figure presents cross-sectional slices at two different distances from the needle tip (20 and $40 \mu \mathrm{m})$ using micro-XCT, nano-XCT and SBFSEM systems. Slices from micro and nano-XCT were manually rotated to spatially align them to the SBFSEM

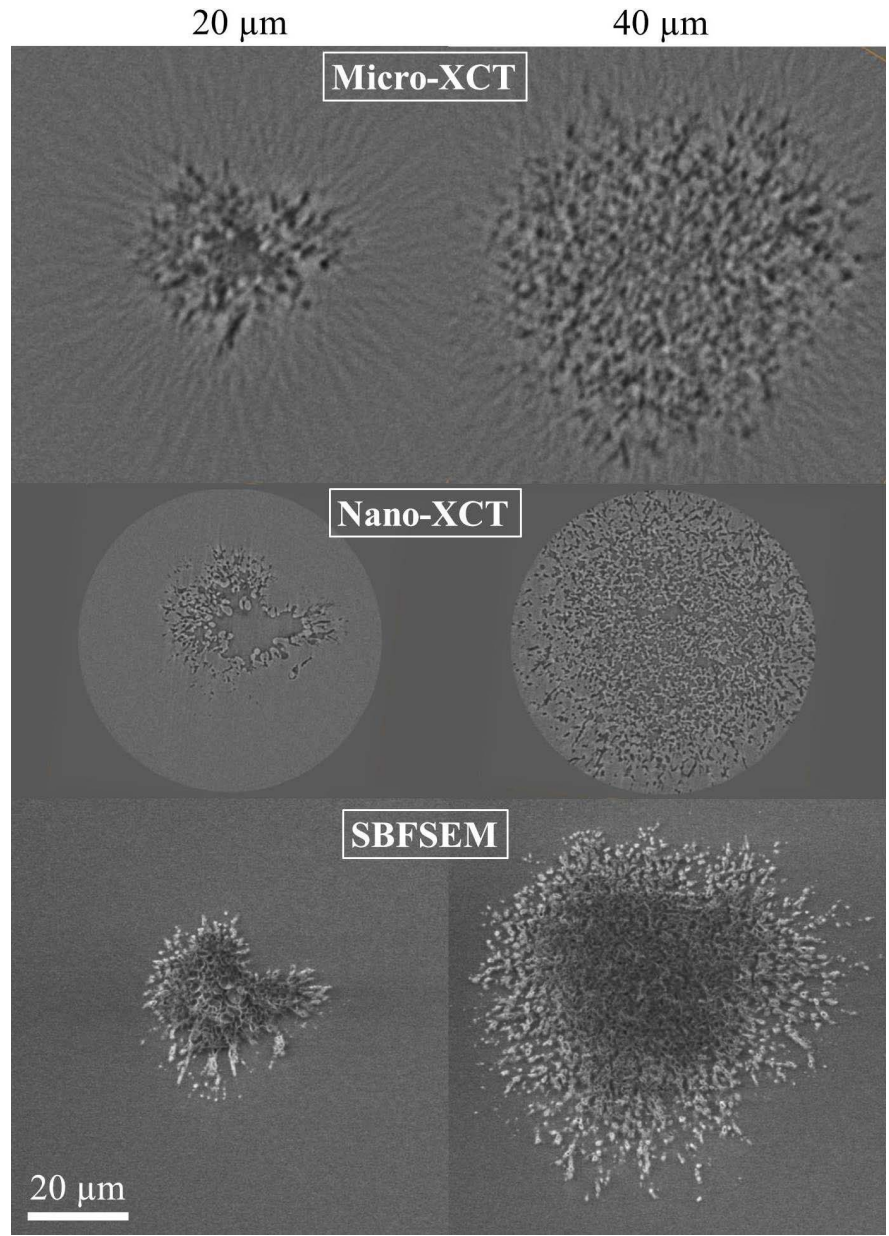

Figure 9. Cross-sections of Sample 4 - Small dense bush tree. Left column: $20 \mu \mathrm{m}$ away from needle tip. Right column: $40 \mu \mathrm{m}$ away.

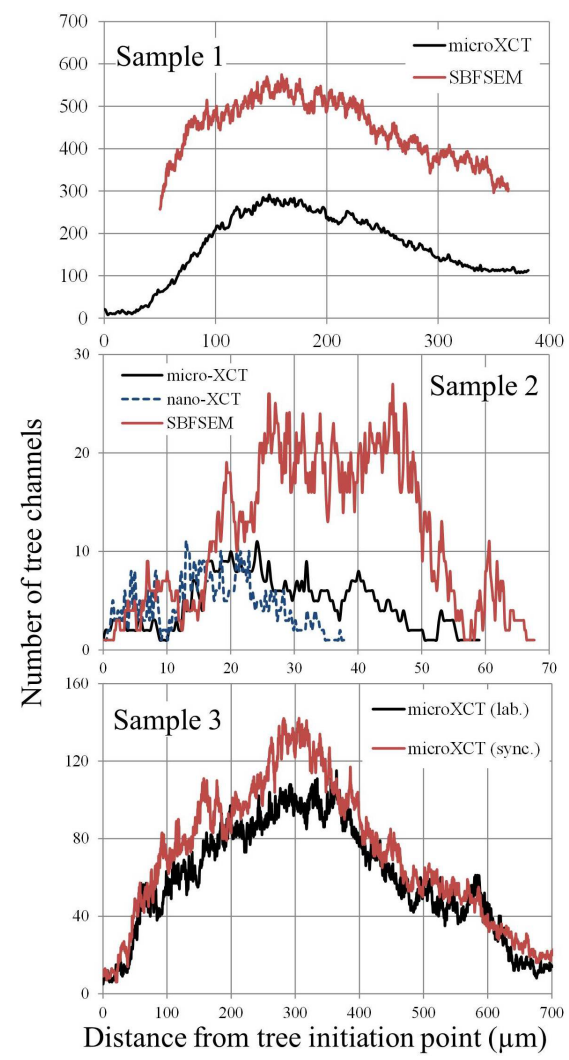

Figure 10. Cross-section analysis: Number of tree channels in each slice as function of the distance from the tree starting point, Samples 1-3. For Sample 1 , only region 2 of SBFSEM data is plotted, as this region corresponds to the XCT data. See Table 2 for the instruments used.

images. Comparing the imaging techniques, it is seen that the features are better defined using nano-XCT and SBFSEM, as expected. Images from the micro-XCT are less clear due to the lower resolution such that individual structures cannot be differentiated so that only the overall damage area can be estimated. Slices from the SBFSEM suggest there are two different areas of degradation which are not clearly distinguished by XCT: an outer, brighter area, where individual channels can be recognized, and an inner-darker area, which is a lower density region and highly degraded. This suggests that the structurally fragile area collapsed during the knife-cutting process of SBFSEM. Nevertheless, the general tree shape is similar regardless of the technique used.

One point of concern in any of these imaging techniques is the observation of branches not connected to the main body of the tree. This can be seen in Figure 6 (XCT - Synchrotron). It is not clear why this happens. It could result from a smaller diameter section that is not resolved, or possibly because the tree tubules have a different nature in that section yielding poor contrast areas and therefore an inability to segment the tree.

\subsection{QUANTITATIVE ANALYSIS 4.2.1 CROSS-SECTIONAL ANALYSIS}

A tree can be analyzed by considering cross-sections (slices) of the tree [7]. The number of channels, the total channel area and the proportion of area degraded, are examples of local parameters that can be calculated in every slice, and used for quantitative comparison of the imaging 
techniques. The progression of the number of tree channels in every cross-sectional slice that is orthogonal to the direction of the needle (' $z$ ' direction as shown in Figure 4) is shown in Figure 10 as a function of the distance from the tree starting point. Sample 4 was excluded from this analysis, since a virtual replica was only created for the micro-XCT scan; for the other techniques image segmentation was difficult either due to uneven contrast or poor signal-to-noise ratio. Furthermore, it is not clear whether the internally degraded region can be considered to consist of discrete channels. Because the SBFSEM procedure failed near to the needle tip for Sample 1 [6], there is some degree of uncertainty as to where the acquired sections are exactly located with respect to the tree initiation point. For this reason, the graph for Sample 1 in Figure 10 has been adjusted in the horizontal axis to match the XCT curve.

It can be observed, that in all the cases presented here, SBFSEM captures a greater number of tree channels than $\mathrm{XCT}$. This is mainly attributed to the smaller pixel size in each slice (see Table 2), but also to a better contrast obtained between the tree channel and the epoxy matrix material, making easier to discriminate or segment tree channels. However, for the bush-type trees (see Samples 1 and 3 in Figure 10), the general trend with distance away from the tree initiation point appears to be similar for all techniques, which was not the case for the branch-type tree.

\subsubsection{GLOBAL PARAMETERS}

Many parameters can be calculated to characterize the entire tree. For example, the diameter of tree channels, volume and surface area of the tree, fractal dimension, number of vertices and segments, segment length, tortuosity and branching angle, among others [7]. The diameter of tree channels and entire volume of the tree are selected for the comparative analysis of the imaging techniques, and are summarized in Table 2. The mean channel diameter is in good correspondence for all the techniques used, with the values from the SBFSEM data sets being generally smaller than $\mathrm{XCT}$. This is attributed to the fact that SBFSEM captures smaller features, decreasing the mean diameter calculated. This is evident when analyzing the frequency distribution of diameters depicted in Figure 11 for Sample 1. For micro-XCT, the minimum diameter of tree channels captured was around $2 \mu \mathrm{m}$, while the SBFSEM data shows that $6 \%$ of channels had diameters below $2 \mu \mathrm{m}$. However, the overall shape of the histograms for the larger vessels is in good agreement with the modal diameters both being between 4-5 $\mu \mathrm{m}$.

The tree volume quantifies the total damaged volume captured, and it provides a better discrimination between the techniques since it is a function of both the volume of specimen imaged as well as the resolution of the technique. In most of the cases, the tree volume captured by SBFSEM is larger than the captured by XCT, as expected considering the better resolution of SBFSEM. A similar verdict can be drawn when comparing the tree length of the replica generated (e.g. see Sample 2 in Table 2), it is clear that SBFSEM captures more of the electrical tree.

Sample 4 was optically seen to be a small and dense bushtype tree of around $105 \mu \mathrm{m}$ length, observations which closely

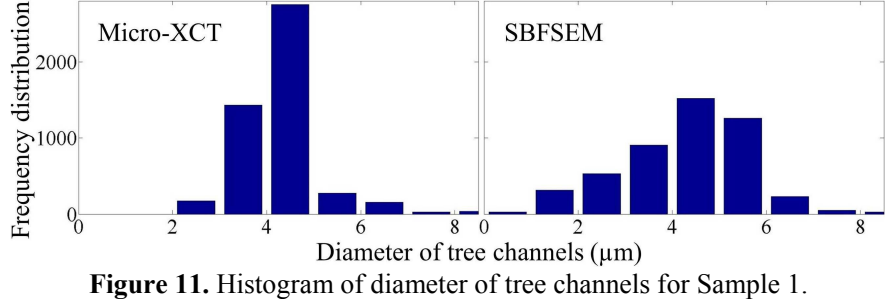

correspond to the 108 and $114 \mu \mathrm{m}$ captured using micro-XCT and SBFSEM, respectively. The 2D projected optical image gives a sense that the shape of the tree is spherical. Assuming that the sphere is completely degraded, it would result in a volume of $6 \times 10^{5} \mu \mathrm{m}^{3}$; however, the virtual replica generated using micro-XCT computed a volume of only $6.7 \times 10^{4} \mu \mathrm{m}^{3}$, or around $10 \%$ of the apparent space. This indicates the limitation of optically analyzing electrical trees in 2D perspective.

\subsection{SAMPLE PREPARATION AND SCAN PROCESS}

As a non-destructive technique, sample preparation for $\mathrm{XCT}$ is much easier than SBFSEM. The sample size required for micro-XCT (2-3 mm) allows multi-stage experiments to be carried out for analyzing tree growth; however, the size required for nano-XCT (under one millimeter) renders it a semi-destructive technique, or at least, it does not allow further electrical stressing without re-embedding the sample in epoxy resin. In both micro and nano-XCT cases the needle serves as a holder and as a reference to locate the tree in the radiographs. It is worth noting the importance of machining the sample as small as possible, to increase the signal-to-noise ratio of the reconstructed images. This is especially important when imaging small features that can be hidden in the background noise of the image. For example, the inability to create a useful virtual replica using nano-XCT for Sample 2 was due to the sample being too large.

For SBFSEM, a block-face area of less than one mm edge without the needle is normally required. The extraction of the needle is carefully performed, but it might cause microfractures around the needle area, yielding uncertainty of the features obtained surrounding the tip. In general, the complexity is greater and the risk of technical problems higher. One of the three cases presented here, where SBFSEM was used, the scan had to be interrupted and sections of the tree lost due to slices been drawn into the final aperture of the instrument or onto the block-face to be imaged. Also, since SBFSEM is destructive, samples can be ruined if the set-up is not the correct at first time. This technique is not compatible with multi-stage experiments.

Another parameter useful for comparison of practical application of the techniques is the scan time. However, the imaging techniques are inherently different in their nature: in $\mathrm{XCT}$, the entire tomography scan is required to reconstruct the slices of the volume imaged, regardless the amount of volume that the feature occupies in the field of view. This can lead to considerable amount of data (slices without useful information) being discarded later. In contrast, in SBFSEM the imaging results are seen immediately, and thus, only the volume of interest is imaged. In the case of laboratory-based $\mathrm{XCT}$, because different devices were used, a considerable 
Table 2. Case studies.

\begin{tabular}{|c|c|c|c|c|c|c|c|c|c|}
\hline Sample & Material & Tree type & Technique / System & Ins trument & $\begin{array}{c}\text { Pixel } \\
\text { size }(\mu \mathrm{m})\end{array}$ & $\begin{array}{c}\text { Scan time } \\
\text { (h) }\end{array}$ & $\begin{array}{c}\text { Length } \\
\text { analyzed } \\
(\mu \mathrm{m})\end{array}$ & $\begin{array}{l}\text { Diameter } \\
\operatorname{(\mu m)}^{(b)}\end{array}$ & $\begin{array}{c}\text { Tree } \\
\text { volume } \\
\left(\mu \mathrm{m}^{3}\right)\end{array}$ \\
\hline \multirow{4}{*}{1} & \multirow{4}{*}{$\begin{array}{l}\text { Flexible } \\
\text { polymer }\end{array}$} & \multirow{4}{*}{ Bush } & micro-XCT (lab) & Xradia Micro-XCT-400 & 1.07 & $54: 30$ & $381^{\text {(c) }}$ & $4.4 \pm 1.7$ & $1.8 \times 10^{6}$ \\
\hline & & & SBFSEM - Region 1 & Gatan 3View-FEI Quanta FEG 250 & 0.2 & 19:06 & 255 & $3.3 \pm 1.5$ & $4.5 \times 10^{6}$ \\
\hline & & & SBFSEM - Region 2 & Gatan 3View-FEI Quanta FEG 250 & 0.2 & $23: 30$ & 313 & $4.5 \pm 2.5$ & $3.9 \times 10^{6}$ \\
\hline & & & Optical camera & & $\sim 4$ & - & $\sim 800$ & - & $2 \times 10^{8(\mathrm{~d})}$ \\
\hline \multirow[t]{2}{*}{2} & \multirow{2}{*}{$\begin{array}{l}\text { Glassy } \\
\text { epoxy }\end{array}$} & \multirow[t]{2}{*}{ Small branch } & micro-XCT (lab) & Zeiss Xradia Versa-510 & 0.371 & $22: 27$ & 59 & $2.0 \pm 0.4$ & $1.6 \times 10^{3}$ \\
\hline & & & SBFSEM & Gatan 3View-FEI Quanta FEG 250 & 0.075 & $7: 13$ & 68 & $1.0 \pm 0.3$ & $1.0 \times 10^{3}$ \\
\hline \multirow{2}{*}{3} & \multirow{2}{*}{$\begin{array}{l}\text { Flexible } \\
\text { polymer }\end{array}$} & \multirow{2}{*}{ Low dens. bush } & micro-XCT (synch.) & I13 - Diamond Light Source UK & 0.365 & $4: 30$ & 840 & $2.7 \pm 1.0$ & $5.0 \times 10^{5}$ \\
\hline & & & micro-XCT (lab) & Zeiss Xradia Versa-510 & 0.454 & $23: 18$ & 720 & $3.1 \pm 1.2$ & $6.5 \times 10^{5}$ \\
\hline 4 & $\begin{array}{c}\text { Flexible } \\
\text { epoxy }\end{array}$ & $\begin{array}{l}\text { Small dense } \\
\text { bush }\end{array}$ & micro-XCT (lab) & Zeiss Xradia Versa-520 & 0.232 & $9: 46$ & 108 & $1.2 \pm 0.3$ & $6.7 \times 10^{4}$ \\
\hline
\end{tabular}

(a) The length was obtained by multiplying the number of slices used to generate the tree by the slice thickness (or voxel size). (b) Diameter calculations: frequency distribution, values presented as 'mean value \pm standard deviation'. (c) Longer tree was captured, but the rest was discarded for calculations, because slices were noisy. (d) Estimation considering tree shape and assuming all tree volume degraded, created with axial rotation of 2D image. (e) No results presented due to the poor quality of the reconstructed slices (bigger sample than required for optimal imaging). (f) Field of view limit. (g) Virtual replica was not generated, due to low signal-to-noise ratio and thus, difficulty in segmentation.

range of scan times was required. Also, scan time greatly depends on sample size (especially for region-of-interest XCT scans) as well as desired resolution and noise level. Thus, it is difficult to carry out clear comparison of scan times. Nevertheless, typical values of scan times can be compared for the practical application of imaging electrical trees.

Imaging the same volume of a mature electrical tree ( 1 $\mathrm{mm}$ length and width), laboratory-based micro-XCT currently requires around $20 \mathrm{~h}$ which is slightly more than with SBFSEM, but with lower resolution. However, individual set-up times are longer using SBFSEM. The fastest technique is synchrotron-based micro-XCT. For the results shown here, micro-XCT at Diamond beamline I13 was around 5 times faster than laboratory micro-XCT. Later, with more experience and improvements in the beamline, scans at this synchrotron, not presented here, were less than $2 \mathrm{~h}$, making it 12-15 times faster than laboratory-based XCT for a comparable pixel size.

\section{SUMMARY}

Two techniques for the 3D imaging of electrical trees, namely, X-ray Computed Tomography (XCT) and Serial Block-Face Scanning Electron Microscopy (SBFSEM) have been extensively trialed. Using these techniques, virtual replicas (3D models) of a variety of electrical trees have been generated and the tree structure analyzed.

SBFSEM is a more direct imaging technique than XCT because every slice is the result of direct SEM imaging. Another consequence of the inherent differences of the imaging techniques is that for XCT the voxel dimensions are symmetric and determined by the pixel size selected during the scan. However, for SBFSEM the voxel dimensions can be asymmetric since the lateral $x y$ pixel size is set by the selected SEM magnification and the vertical $z$ pixel size is determined by the chosen slice thickness. This allows the selection of a finer lateral resolution in cross-sections of the tree where required. Another key distinction is that in XCT, selecting a finer pixel size results in a smaller field of view, while in SBFSEM the field of view (or area to be scanned) is independent of the pixel size selected. Thus, in SBFSEM, relatively large volumes can be captured with high resolution, while in XCT higher resolutions are achievable only by limiting the volume captured (and the sample size too) [24].

Overall, SBFSEM provides greater detail than XCT as expected. This is evident not only when comparing the visual aspect of the original cross-sectional images or the virtual replicas generated, but also quantitatively comparing parameters such as number of tree channels and tree volume captured. This greater detail is mainly attributed to the finer resolution of the technique. Currently for the case of polymeric materials, the lateral resolution is around $20 \mathrm{~nm}$, and the minimum slice thickness (vertical resolution) is practically around 20-30 nm (though technically the SBFSEM machine can slice as thin as 5 $\mathrm{nm})$. In this application of imaging electrical trees, the smallest feature that was possible to resolve was around $0.2-0.3 \mu \mathrm{m}$. A smaller pixel size would yield more detailed data in each image, however, when are handled in a stack of thousands, the computational process becomes more difficult and time consuming. Consequently, SBFSEM is recommended for detailed imaging of sections of the tree, for example, tree tips or early tree growth. SBFSEM is not the most suitable technique for imaging an entire mature electrical tree, due to its destructive nature and the possibility of slices been drawn into the final aperture of the instrument or onto the block-face to be imaged. Also, SBFSEM is not appropriate for imaging a highly degraded 
region, as the matrix becomes structurally weak and vulnerable to slicing damage. Using the instrumentation deployed here, the block face to be imaged could be no bigger than around $800 \mu \mathrm{m}$, due to the limited cutting window of the knife.

In the case of XCT, currently the finest voxel size is around $0.3 \mu \mathrm{m}$ for micro-XCT (with a field of view of around $0.5 \mathrm{~mm}$ by $0.5 \mathrm{~mm}$ ) and around $16 \mathrm{~nm}$ for nano$\mathrm{XCT}$, but with a field of view of only around $15 \mu \mathrm{m}$ by 15 $\mu \mathrm{m}$. However, in practice, the final spatial resolution for $\mathrm{XCT}$ can be limited by the source spot size and modulation transfer function of the detector, as well as the noise in the data. As such, the resolution may be a few times greater than the voxel size. Consequently, micro-XCT allows imaging the structure of a relatively medium to mature electrical tree (hundreds of micrometers), but does not satisfactorily resolve features smaller than $\sim 0.7 \mu \mathrm{m}$. Using nano-XCT, features of the order of hundreds of nanometers can be resolved and accurately characterized. This makes nano-XCT suitable for early tree growth studies, but not for more mature trees. In summary, for imaging a medium to large size electrical tree, the recommended technique is micro-XCT which allows larger volumes $\left(\sim 1 \mathrm{~mm}^{3}\right)$ to be 3D imaged with a reasonable voxel size of $\sim 0.3 \mu \mathrm{m}$, but this does compromise the capture of fine details smaller than $\sim 0.5 \mu \mathrm{m}$. Typical scan times are around $20 \mathrm{~h}$ for laboratory-based XCT, $2 \mathrm{~h}$ for synchrotron-based XCT and 10-20 $\mathrm{h}$ for SBFSEM, depending on the volume to be imaged.

\section{CONCLUSION}

Optical methods are appropriate for monitoring tree growth by providing a fast, real-time efficient overall view of the treeing structure. However, optical imaging is limited to translucent materials and only provides 2D projected images of the tree, with a resolution around tens of microns. XCT and SBFSEM can provide the full 3D structure of a tree, which is a radical improvement over a 2D optical projection. An important benefit of using these $3 \mathrm{D}$ imaging techniques is the ability to internally explore complex dense structures such as bush-type electrical trees. Another benefit is the possibilities of analysis and quantification that the $3 \mathrm{D}$ data brings.

Using micro-XCT, fine details of the tree (tree tips and small sub-branches) are lost, but an entire mature tree $(\sim 1$ $\mathrm{mm}$ length) can be captured and the technique's nondestructive nature allows multi-stage experiments to be conducted and thus facilitating the analysis of tree growth. SBFSEM provides the highest resolution of the techniques analyzed here; however the destructive nature of the procedure makes the process less reliable than XCT. Care must also be taken with SBFSEM that the associated microtome technique does not alter the tree features.

Virtual replicas generated using both XCT and SBFSEM are consistent with each other and to optical images. The greater level of detail rendered by SBFSEM is not only noticed by visual inspection of the $3 \mathrm{D}$ models, but also comparing quantitative parameters. On average, SBFSEM captures almost double the number of tree channels per slice than XCT, while virtual replicas in most of the cases have larger volumes, which indicate that more of the electrical tree is captured.

It is proposed that for full analysis a combination of imaging techniques is the appropriate methodology. Optical methods are used first to monitor tree growth and have an initial idea of size and structure of the tree. Then, the micro-XCT technique, which provides pixel size $\sim 0.4$ $\mu \mathrm{m}$ with a field of view of around $1 \mathrm{~mm} \times 1 \mathrm{~mm}$, can be used to reveal the entire $3 \mathrm{D}$ structure of a normal/mature electrical tree. As XCT is non-destructive, scan parameters can be tuned to optimize the imaging results. Moreover, the same sample can be analyzed at different stages of tree growth. Nano-XCT can be used to explore in more detail regions of interest highlighted by the micro-XCT data, with a pixel size $\sim 65 \mathrm{~nm}$, but a limited field of view of $65 \mu \mathrm{m}$. Finally, sections of the tree can be analyzed in even more detail using SBFSEM (again under guidance from micro-XCT data), which can provide resolutions below $50 \mathrm{~nm}$. Finally, it would be possible to analyze an interesting block-face with even higher resolution, by gold-coating its surface and using conventional SEM. In most instances a subset of these techniques will be appropriate and necessary to control costs and efficiency.

\section{ACKNOWLEDGMENT}

This work was partially funded by the RCUK's Energy Programme through the Top \& Tail Transformation programme grant, EP/I031707/1 (http://www.topandtail.org.uk/). Roger Schurch would like to acknowledge the scholarship support of CONICYT (Chilean Research Council). Philip Withers would like to acknowledge funding from EPSRC for the Manchester X-ray Imaging Facility under EP/F007906/1 and EP/F028431/1. Robert Bradley would like to acknowledge funding from Carl Zeiss XRM. The authors thank Dr. Tobias Starborg in the EM facility at the Faculty of Life Sciences in the University of Manchester for his assistance, and the Wellcome Trust for equipment grant support to the EM facility.

\section{REFERENCES}

[1] R. Schurch, S. M. Rowland, R. S. Bradley, and P. J. Withers, "Imaging and analysis techniques for electrical trees using X-ray computed tomography", IEEE Trans. Dielectr. Electr. Insul., Vol. 21, pp. 53-63, 2014.

[2] C. Xiangrong, X. Yang, C. Xiaolong, S. J. Dodd, and L. A. Dissado, "Effect of tree channel conductivity on electrical tree shape and breakdown in XLPE cable insulation samples", IEEE Trans. Dielectr. Electr. Insul., Vol. 18, pp. 847-860, 2011.

[3] A. S. Vaughan, I. L. Hosier, S. J. Dodd, and S. J. Sutton, "On the structure and chemistry of electrical trees in polyethylene", J. Phys. D: App. Phys., Vol. 39, p. 962, 2006.

[4] C. Yu, T. Imai, Y. Ohki, and T. Tanaka, "Tree initiation time evaluation of epoxy/silica composites by partial discharge detection", IEEE Int'l. Conf. Prop. Applic. Dielectr. Materials (ICPADM), pp. 404-407, 2009.

[5] N. Hozumi, M. Ishida, T. Okamoto, and H. Fukagawa, "The influence of morphology on electrical tree initiation in polyethylene under $\mathrm{AC}$ and impulse voltages", IEEE Trans. Electr. Insul., Vol. 25, pp. 707-714, 1990. 
[6] R. Schurch, S. M. Rowland, and T. Starborg, "Serial Block-Face Scanning Electron Microscopy for Three- Dimensional Imaging of Electrical Trees", IEEE Int'l. Conf. Solid Dielectr. (ICSD), pp. 271274, 2013.

[7] R. Schurch, S. M. Rowland, R. S. Bradley, and P. J. Withers, "Three dimensional characterisation of electrical trees", IEEE Conf. Electr. Insul. Dielectr. Phenomena (CEIDP), pp. 494-497, 2013.

[8] N. Menn, Practical optics, Academic Press, 2004.

[9] G. H. Michler, Electron microscopy of polymers, Springer, 2008.

[10] A. C. Kak and M. Slaney, Principles of Computerized Tomographic Imaging, Society for Industrial and Applied Mathematics, 2001.

[11] P. Cloetens, M. Pateyron-Salomé, J. Y. Buffière, G. Peix, J. Baruchel, F. Peyrin, et al., "Observation of microstructure and damage in materials by phase sensitive radiography and tomography", J. App. Phys., Vol. 81, pp. 5878-5886, 1997.

[12] R. S. Bradley, A. McNeil, and P. J. Withers, "An examination of phase retrieval algorithms as applied to phase contrast tomography using laboratory sources", Proc. SPIE, Developments in X Ray Tomography, San Diego, California, USA, pp. 780404-10, 2010.

[13] P. Cloetens, R. Barrett, J. Baruchel, J. P. Guigay, and M. Schlenker, "Phase objects in synchrotron radiation hard x-ray imaging", J. Phys. D: App. Phys., Vol. 29, pp. 133-146, 1996.

[14] K. L. Kelly and B. K. Tanner, "Factors affecting in-line phase-contrast imaging with a laboratory micro-focus X-ray source", Advances in Xray Analysis, Vol. 49, pp. 31-36, 2006.

[15] P. J. Withers, "X-ray nanotomography", Materials Today, Vol. 10, pp. 26-34, 2007.

[16] A. Tkachuk, F. Duewer, H. Cui, M. Feser, S. Wang, and W. Yun, "Xray computed tomography in Zernike phase contrast mode at $8 \mathrm{keV}$ with $50-\mathrm{nm}$ resolution using $\mathrm{Cu}$ rotating anode X-ray source", Zeitschrift fur Kristallographie, Vol. 222, pp. 650-655, 2007.

[17] R. Mokso, F. Marone, and M. Stampanoni, "Real Time Tomography at the Swiss Light Source", Proc. AIP Conf., Vol. 1234, pp. 87-90, 2010.

[18] A. M. Donald, "The use of environmental scanning electron microscopy for imaging wet and insulating materials", Nature Materials, Vol. 2, pp. 511-516, 2003.

[19] R. F. Egerton, Physical principles of electron microscopy: an introduction to TEM, SEM, and AEM, Springer, 2005.

[20] W. Denk and H. Horstmann, "Serial Block-Face Scanning Electron Microscopy to Reconstruct Three-Dimensional Tissue Nanostructure", PLoS Biol, Vol. 2, pp. 1900-1909, 2004.

[21] G. E. Thompson, T. Hashimoto, X. L. Zhong, M. Curioni, X. Zhou, P. Skeldon, et al., "Revealing the three dimensional internal structure of aluminium alloys", Surface and Interface Analysis, Vol. 45, pp. 1536$1542,2013$.

[22] R. S. Bradley and J. P. Withers, "Analysis of 3-D networks with surface texture from volumetric image data using sphere-networks", Submitted to IEEE Trans. Pattern Analysis and Machine Intelligence, 2014.

[23] R. Schurch, S. M. Rowland, R. S. Bradley, and P. J. Withers. "Electrical Trees CEIDP", (Accessed on 2013, $11^{\text {th }}$ June). Available: http://www.youtube.com/user/ElectricalTreesCEIDP/videos

[24] E. Maire and P. J. Withers, "Quantitative X-ray tomography", International Materials Reviews, Vol. 59, pp. 1-43, 2014.

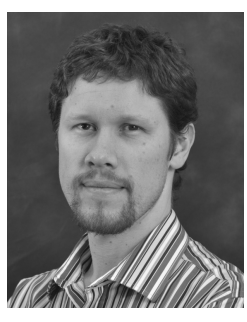

Roger Schurch (S'11) was born in Temuco, Chile. $\mathrm{He}$ received the degree in electrical engineering in 2006 from Federico Santa Maria Technical University (UTFSM), Valparaiso, Chile. He was a high voltage equipment analyst at Transelec transmission company in Santiago, Chile, from 2006-08. He joined the Department of Electrical Engineering at UTFSM, as a Junior Lecturer in 2008, where he also carried out dielectric tests for mining and utility companies. Since 2011, he has been a PhD student at the School of Electrical and Electronic Engineering in The University of Manchester. His research project involves the study of electrical trees and partial discharges in polymeric insulation. He was awarded the IEEE Graduate Fellowship of the Dielectrics and Electric Insulation Society in 2011.

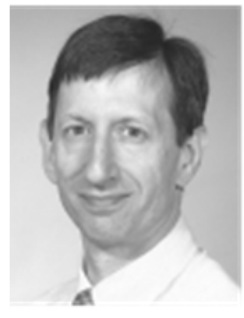

Simon M. Rowland (F'14) was born in London, England. He completed the B.Sc. degree in physics at The University of East Anglia and the Ph.D. degree at London University. He has worked for many years on dielectrics and their applications and has also been Operations and Technical Director within multinational manufacturing companies. He joined The School of Electrical and Electronic Engineering in The University of Manchester as a Senior Lecturer in 2003, and was appointed Professor of Electrical Materials in 2009. He was President of the IEEE Dielectric and Electrical Insulation Society from 2011-12.

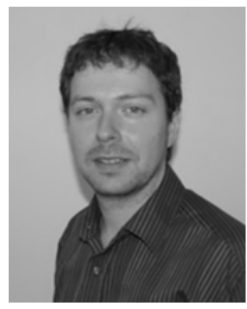

Robert S. Bradley was born in St Asaph, UK. He completed BA and MSc degrees in Naturals Sciences (physics) at Cambridge University before gaining a Ph.D. in fluorescence imaging at The University of Manchester. He has since worked as a Postdoctoral Research Associate in Positron Emission Tomography at the Wolfson Molecular Imaging Centre before joining the School of Materials at The University of Manchester in 2008 to help with the setting up of the Henry Moseley X-ray Imaging Facility. He is currently a Zeiss Applications Fellow and his ongoing research interests include the development and application of phase contrast and 4-D nano-scale X-ray tomography.

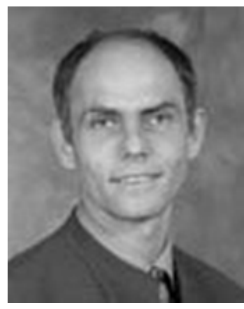

Philip J. Withers was born in Usk, Wales. He completed his BA in Natural Sciences at Cambridge specializing in Theoretical Physics in 1985 and his $\mathrm{PhD}$ in 1989 in Materials Science at Cambridge. After 9 years as a Lecturer in Cambridge he took up a Chair in Materials Science at Manchester University in 1998. His work has focused on understanding the performance and structural integrity of engineering materials and components, with particular focus on the effect of residual stresses. In 2009 he set up the Henry Moseley X-ray Imaging Facility at Manchester which has some of the best facilities for computer tomographic imaging of materials in the UK. He is now also Director of the BP International Centre for Advanced Materials. 